\title{
DENGLISCHE WERBESPRACHE - EINE UMFRAGE ZUM VERSTEHEN VON ANGLIZISMEN IN DER DEUTSCHEN SPRACHE
}

Jede lebende Sprache, darunter auch die deutsche, ist einem ständigen Wandel unterzogen und die Aufnahme von Wörtern aus allen Teilen der Welt gehört unabdingbar dazu. Gegenwärtig dominiert das aus der englischen Sprache stammende Lehngut im deutschen Sprachgebrauch. Dieses Phänomen findet Interesse sowohl im öffentlichen Diskurs als auch in der linguistischen Forschung. Viele Deutsche kommen kaum noch ohne Englisch aus. Es ist normal, alltäglich und gewöhnlich geworden. Anglizismen finden wir in allen Bereichen des Deutschen und sie werden nach wie vor zu einem festen Bestandteil des deutschen Wortschatzes. Der vermehrte Gebrauch von Anglizismen führt, insbesondere in der Werbesprache, zu einer Mischung der zwei Sprachen Deutsch und Englisch und zur Entstehung eines neuen Sprachenmix „Denglisch“ ${ }^{\circ 2}$, der sowohl in der geschriebenen als auch in der gesprochenen Sprache $\mathrm{zu}$ finden ist.

Im vorliegenden Artikel wird untersucht, ob und wie Sprecher des Deutschen englische Werbeslogans verstehen. Zuvor werden die zentralen Begriffe, nämlich Anglizismen, Denglisch und Werbeslogan, diskutiert. Danach werden die deutschen Werbepresseslogans unter der Berücksichtigung der denglischen Werbesprache und der meistgebrauchten Werbewörter präsentiert. Zum Schluss werden die Ergebnisse der empirischen Untersuchung dargestellt, die in Deutschland in zwei Altersgruppen durchgeführt wurde. Sie unterscheiden sich von den Ergebnissen, die die Süddeutsche Zeitung im Jahre 2009 durchgeführt hat.

Schlüsselwörter: Denglisch, Sprache, Anglizismen, Werbeslogan, Werbung.

\section{HINFÜHRUNG ZUM THEMA}

In der letzten Zeit wird viel über den Einfluss von Anglizismen auf die deutsche Sprache diskutiert, weil die Zahl der Anglizismen in der deutschen Sprache von Jahr zu Jahr zunimmt. Dieser Einfluss war und ist so intensiv, dass eine Mischung aus zwei Sprachen entstanden ist. Diese Mischung aus Deutsch und Englisch wird als „Denglisch ${ }^{63}$ bezeichnet und ist sowohl in der geschriebenen als auch in der gesprochenen Sprache zu finden.

Bereits seit dem Jahr 2000 kann man in vielen deutschen Fachsprachen und Lebensbereichen einen wesentlichen Einfluss vom Englischen auf das Deutsche beobachten. ${ }^{4}$

Dieser Beitrag widmet sich der Textsorte der Werbeslogans. Es wird überprüft, wie weit diese Erscheinung in den letzten Jahren fortgeschritten ist und ob sie von den Deutschen als positiv oder eher negativ empfunden wird.

\footnotetext{
${ }^{1}$ Mag. Agnieszka Czech-Rogoyska, Fremdsprachenzentrum der Rzeszower Universität, Prof. St. Pigonia Straße 1, Gebäude A0/B2, Zi.79, 35-959 Rzeszow, mail: a.rogoyska@gmail.com, (Uniwersyteckie Centrum Nauki Języków Obcych, Uniwersytet Rzeszowski, ul. Prof. St. Pigonia 1, budynek A0/B2, pokój 79, 35-959 Rzeszów).

${ }^{2}$ Vgl. Duden - Deutsches Universalwörterbuch, Mannheim 2001, S. 388.

${ }^{3}$ Vgl. ibidem, S. 388

${ }^{4}$ Vgl. A. Czech-Rogoyska, Denglisch - czas anglicyzmów w języków niemieckim, „Lingua legis” 2002/10, S. 53-63.
} 


\section{DEFINITORISCHE VERSUCHE}

Zunächst muss geklärt werden, was unter den Begriffen Anglizismus, Denglisch und Werbeslogan $\mathrm{zu}$ verstehen ist.

\subsection{Anglizismus}

In einer Vielzahl von Untersuchungen zum Thema Anglizismen wird zunächst von Horst Zindlers Definition ausgegangen: „Ein Anglizismus ist ein Wort aus dem britischen oder amerikanischen Englisch im Deutschen oder eine nicht übliche Wortkomposition, jede Art der Veränderung einer deutschen Wortbedeutung oder Wortverwendung (Lehnbedeutung, Lehnübersetzung, Lehnübertragung, Lehnschöpfung, Frequenz-Steigerung, Wiederbelebung) nach britischem oder amerikanischem Vorbild“5 Diese Definition hat durch Broder Carstensen eine Erweiterung im Hinblick auf die Laut-, Satz- und Textebene sowie die Differenzierung zwischen Britizismen und Amerikanismen erfahren. ${ }^{6}$

Bei einem Anglizismus handelt es sich um ein Wort, das aus der englischen Sprache in den Wortschatz einer anderen eingegangen ist. Als Anglizismus bezeichnet man eine Übernahme oder Entlehnung aus allen Varietäten des Englischen ins Deutsche. Bei diesen Einflüssen des Englischen auf das Deutsche wird die Wortkreuzung „Denglisch“ verwendet.

\subsection{Denglisch}

Die meisten Definitionen des Begriffs „Denglisch“ schildern diese Erscheinung als eine neue Sprache, die infolge einer Mischung von zwei Sprachen, also Deutsch und Englisch, entstanden ist. Neben der Bezeichnung Denglisch kann man auch andere Begriffe für dieses Phänomen finden, nämlich Denglish, Engleutsch und Germish. Denglisch ist ein relativ neuer, aber legitimer und integrierter Bestandteil der deutschen Sprache.

Bei dieser Sprachmischung oder Mixsprache handelt es sich also um Wortschöpfungen, die aus deutschen und englischen Begriffen zusammengesetzt wurden.

Denglisch besteht aus Wörtern bzw. Phrasen wirklich oder scheinbar englischer Herkunft und umfasst

- Lehnwörter und -phrasen aus der englischen Sprache, d.h. Anglizismen wie Airbag, Coffee to go, Download, Player, Shuttle, Bodyguard, Chip;

- Wörter, die zwar mehr oder weniger englisch geschrieben und ausgesprochen werden, aber von englischen Muttersprachlern nicht bzw. mit anderer Bedeutung verwendet werden, d.h. Pseudo-Anglizismen wie Handy, Oldtimer, Beamer, Dressman, Evergreen, Quizmaster, checken;

- Phrasen aus deutschen Wörtern, die es 1:1 übersetzt auch im Englischen gibt, d.h. angebliche oder wirkliche Lehnübersetzungen wie macht Sinn, nicht wirklich, ich denke, am Ende des Tages. ${ }^{7}$

\footnotetext{
5 H. Zindler, Anglizismen in der deutschen Pressesprache nach 1945, Diss. Kiel 1959, S. 2. Zitiert nach: U. Busse, Anglizismen im Duden. Eine Untersuchung zur Darstellung englischen Wortguts in den Ausgaben des Rechtschreibdudens von 1880-1986, Tübingen 1993, (Reihe Germanistische Linguistik 139), S. 15.

${ }^{6}$ Vgl. B. Carstensen, Englische Einflüsse auf die deutsche Sprache nach 1945, Heidelberg 1965, (Beihefte zum Jahrbuch für Amerikastudien 13), S. 30.

${ }^{7}$ Vgl. Denglisch 4ever, http://www.denglisch4ever.de/autoframes.html?/top10.html (Zugriff am 18.04.2014).
} 


\subsection{Werbeslogan}

Viele Werbetexte enthalten Slogans. Der Slogan ist auch als wichtigster Textbestandteil einer Werbeanzeige zu betrachten. Werbeslogans bieten einen sehr guten Zugriff auf die Spezifika der Werbesprache. Man versteht unter einem Slogan „eine formelhaft kurze, graphisch (oder sprecherisch) und bedeutungsmäßig meist isoliert erscheinende Zeile, die in der Regel längere Zeit benutzt wird“8 . Nach Anke Wächter sollten Werbeslogans die Werbeaussage und den Produktnamen in das Bewusstsein des Empfängers „eingraben“. Die Einprägsamkeit des Slogans unterstützen die inhaltliche Prägnanz und die Originalität der Gestaltung und deswegen sei hier mit einer intensiven Ausnutzung der sprachlichen Formen und Strukturen zu rechnen. ${ }^{9}$

\section{DEUTSCHE WERBESLOGANS IN DEN MEDIEN}

Viele der Ausländer, die nach Deutschland kommen und nur Englisch sprechen, haben keine Probleme mit dem Verstehen der deutschen Werbeslogans in den Medien, da diese viele englische Wörter beinhalten. Diese Situation macht die zunehmende Verwendung von Anglizismen in der deutschen Sprache sehr deutlich.

\subsection{Denglisch in der deutschen Werbung}

Die Anzahl der in englischer Sprache abgefassten Werbeanzeigen, mit denen Millionen Deutsche jeden Tag konfrontiert werden, nimmt ständig zu. Die Deutschen beobachten solche Sprachveränderungen oft mit Besorgnis und immer häufiger vertreten sie den Standpunkt, dass die deutsche Sprache von Anglizismen überschwemmt wird. In diesem Zusammenhang hat man es nicht mehr mit Deutsch zu tun, sondern mit Denglisch. ${ }^{10}$

Die deutsche Werbung bietet Hits for Kids oder Joghurt mit Weekend Feeling. Im deutschen Fernsehen gibt es den Kiddie Contest, History, Adventure oder History Specials und im Radio Romantic Dreams. Die Deutschen stählen ihren Körper mit Body Shaping und Power Walking. Sie kleiden sich in Outdoor Jack, Tops oder Beach Wear. Sie pflegen sich mit Anti-Aging-Creme oder sprühen Styling ins Haar. Bei der Bahn mit ihren Tickets, dem Service Point und McClean verstehen sie oft leider nur Bahnhof. $^{11}$

Die Untersuchung der Häufigkeit des Vorkommens von Anglizismen zeigt, dass die „Bild“-Zeitung den höchsten Anteil an englischen Entlehnungen aufweist. Besonders auffällig ist der Unterschied zur „Frankfurter Allgemeinen Zeitung“, für die sich nur ein wenig mehr als ein Drittel der Anglizismenzahl der „Bild“-Zeitung“" ergibt. ${ }^{12}$

\footnotetext{
${ }^{8}$ Vgl. B. Sowinski, Werbung, Tübingen 1998, S. 59.

${ }^{9}$ Vgl. A. Wächter, Deutsche Sprache im 18. Jahrhundert-wie spiegeln sich kommunikative Verhältnisse in den Stillehrbüchern dieser Zeit wider?, Institut für Deutsche Sprache, Mannheim 1992, S. 89.

${ }^{10} \mathrm{Vgl}$. K. Meder, Anglizismen in der deutschen Werbesprache: Untersucht anhand ausgewählter Frauen- und Männerzeitschriften, Berlin 2006.

11 Vgl. Verein Deutsche Sprache, Denglisch. Deutsch oder Denglisch?, http://www.vds-ev.de/ag-denglischthema (Zugriff am 23.03.2013).

${ }^{12}$ Vgl. M. Adler, Form und Häufigkeit der Verwendung von Anglizismen in deutschen und schwedischen Massenmedien, 2., neu bearbeitete Fassung, Jena 2004, http://www.db-thueringen.de/servlets/ DerivateServlet/Derivate-3386/Adler.txt (Zugriff am 23.03.2013).
} 


\subsection{Meistgebrauchte Werbewörter in den deutschen Werbeslogans}

Unter den meistgebrauchten Wörtern in deutschen Werbeslogans kann man auch Anglizismen finden. An erster Stelle dieser Rangliste steht das Wörtchen „wir“", gefolgt von dem Substantiv „Leben“. Den dritten Platz behauptet „mehr“, es folgen „einfach“ und „Sie“. An sechster Stelle landet das englische Pronomen „your". „Hier“, ,ich“, „natürlich“ und ,gut" bilden die hinteren vier Plätze der zehn meistgebrauchten Werbewörter in den deutschen Werbeslogans. ${ }^{13}$ Das Wort „we $\mathbf{e}^{6}$ rangiert unter den 15 meistverwendeten Begriffen in deutschen Claims.

Man kann beobachten, dass die Werbetexter auf Wir-Gefühl, Englisch und Kunstwörter setzen. Die englische Sprache gilt gegenwärtig als internationales Bindeglied.

\section{3. „WORST CASE IST KEINE WURSTKISTE“14}

Sind englische Werbeslogans für die Deutschen verständlich? Als Titel zu diesem Abschnitt meines Artikels hat mir der Titel des Artikels über die denglische Werbung „Worst Case ist keine Wurstkiste“"15 gedient, der auch als ein gutes Beispiel für denglische Werbeslogans dienen könnte.

Mit dieser Frage beschäftigen sich seit Jahren viele Forscher und Wissenschaftler ${ }^{16}$. Einerseits lieben die Deutschen die englische Sprache ${ }^{17}$ und verwenden ständig Englisch in der Alltags- und Berufssprache, andererseits sind Anglizismen in der Werbung deutschen Konsumenten oft nicht nur unverständlich, sondern lassen sie auch kalt. ${ }^{18}$ Viele Sprachschützer hoffen, dass die Anglizismen in der Zukunft aus der Werbung und sogar aus der gesamten deutschen Sprache verschwinden. Dieses geschieht aber heutzutage nicht, im Gegenteil, die deutsche Sprache verfalle und die Deutschen sprechen Denglisch, so ist eine verbreitete Meinung. ${ }^{19}$

Besonders die Werbung sei von Anglizismen überflutet. ${ }^{20}$ „Englisch ist die beliebteste Sprache der Reklameprofis“", ${ }^{21}$ war schon 2003 im Spiegel zu lesen.

Sollten also die deutschen Werbespots mit Hilfe von Anglizismen verfasst werden? Aus vielen Untersuchungsergebnissen geht hervor, dass die Anglizismen und Denglisch in

13 Vgl. L. Herrmann, Die zehn meistgebrauchten Werbewörter; (Quelle: Slogometer), http://www.wuv.de/ marketing/die_zehn_meistgebrauchten_werbewoerter(Zugriff am 20.02.2013).

${ }^{14}$ Vgl. Spiegel online 2011, Denglische Werbung: Worst Case ist keine Wurstkiste, http://www.spiegel.de/karriere/berufsleben/denglische-werbung-worst-case-ist-keine-wurstkiste-a760476.html (Zugriff am 12.11.2012).

${ }^{15} \mathrm{Vgl}$. ibidem.

${ }^{16}$ Vgl. S. Burmasova, Empirische Untersuchung der Anglizismen im Deutschen am Material der Zeitung Die WELT (Jahrgänge 1994 und 2004), Bamberger Beiträge zur Linguistik, University of Bamberg Press, Bamberg 2010, S. 10-11.

${ }^{17}$ Vgl. Spiegel online 2011, op. cit.

${ }^{18} \mathrm{Vgl}$. Spiegel online 2004, http://www.spiegel.de/unispiegel/wunderbar/denglisch-in-der-werbung-komm-reinund-finde-wieder-raus-a-310548.html (Zugriff am 12.11.2012).

${ }^{19} \mathrm{Vgl}$. Spiegel online. Wirtschaft, Werbung wieder deutsch, "Überleben Sie die Fahrt in unserem Auto", 2004, http://www.spiegel.de/wirtschaft/werbung-wieder-deutsch-ueberleben-sie-die-fahrt-in-unserem-auto-a287098.html (Zugriff am 12.11.2012).

${ }^{20} \mathrm{Vgl}$. J.K. Androutsopoulos, Sprachwahl im Werbeslogan. Zeitliche Entwicklung und branchenspezifische Verteilung englischer Slogans in der Datenbank von slogans.de, Hannover 2004, S. 4, http://www.mediensprache.net/networx/networx-41.pdf (Zugriff am 12.11.2012).

${ }^{21}$ T. Tuma, Die Sense stimuliert, ,Spiegel” 2003/38, S. 87. 
den deutschen Werbetexten den Deutschen viele Verständnisprobleme bereiten. Einige seien im Folgenden skizziert.

Eine der Untersuchungen hat die Dortmunder Statistikerin Isabel Kick durchgeführt. ${ }^{22}$ Kick testete in einer Studie zum Thema Denglisch die Reaktion ihrer Probanden auf verschiedene Texte in der Werbung, indem sie den Hauptwiderstand beim Anhören der einzelnen Slogans maß. Dabei stellte sich heraus, dass die Gefühlsreaktionen bei Werbesprüchen auf Deutsch, wie zum Beispiel „Geiz ist Geil“, „Wir sind da“, oder „Ganz schön clever“, deutlich höher ausfielen als bei Sprüchen wie „Come in and find out“ oder „Designed to make a difference".

Als ausschlaggebenden Grund für dieses Ergebnis vermutete Kick einfach Verständnisprobleme bei den Spots auf Denglisch. Auch schaffe es Werbung mit englischen Slogans kaum, ausreichend Aufmerksamkeit zu erregen. ${ }^{23}$

Mit dem Phänomen „Denglisch“ in der deutschen Werbung hat sich auch der Verein der Deutschen Sprache mit Walter Krämer als Vorsitzenden beschäftigt. ${ }^{24}$ Dieser ist natürlich erfreut über das Ergebnis der Studie der Dortmunderin Isabel Kick. „Ein alberner Anglizismus und eine ärgerliche Flucht aus der deutschen Sprache“, zürnt der Vizevorsitzende des Vereins der Deutschen Sprache Gerd Schrammen, „Engländer und Amerikaner lachen sich kaputt über den deutschen Drang zum Englischen." 25

Immerhin hat aber der Verein auch festgestellt, dass der Trend in der Werbung wieder weg vom hässlichen „Denglisch“ geht. McDonalds beispielsweise änderte seinen Slogan von „Every time a good time“ zu „Ich liebe es“.

Die Art und Weise, wie englische Begriffe in die deutsche Sprache aufgenommen werden, ist für die Deutschen manchmal peinlich. Viele Formulierungen sind einfach missverständlich. In der „Bild“ ist zu lesen, dass die deutschen Werbetexte mit vielen denglischen Formulierungen verwirrend seien und oft in eine völlig andere Richtung als ursprünglich gewollt gingen. ${ }^{26}$ Weiter lesen wir in der „Bild“ ${ }^{27}$, die Werbetexter wollten, dass die Reklame von den Konsumenten nicht nur verstanden, sondern auch cool empfunden werden sollte.

\section{DARSTELLUNG DER EMPIRISCHEN UNTERSUCHUNGEN}

Für die Umfrage werden folgende Fragen zugrunde gelegt:

1) Hängt das Verständnis des Werbeslogans von Alter und Schulbildung ab?

2) Für welche Zielgruppen eignen sich besonders englische Slogans?

\footnotetext{
${ }^{22}$ Vgl. I. Kick, Die Wirkung von Anglizismen in der Werbung. „Just do it“ oder lieber doch nicht?, IFB Verlag, Paderborn 2004.

${ }^{23}$ Vgl. J. Leffers, Denglisch in der Werbung: Komm rein und finde wieder raus, Spiegel online. Unispiegel. 2004, http://www.spiegel.de/unispiegel/wunderbar/denglisch-in-der-werbung-komm-rein-und-finde-wiederraus-a-310548.html (Zugriff am 20.02.2013).

${ }^{24}$ Vgl. K. Wirth, Der Verein Deutsche Sprache. Hintergrund, Entstehung, Arbeit und Organisation eines deutschen Sprachvereins. Bamberger Beiträge zur Linguistik, University of Bamberg Press, Bamberg 2010, S. $157-163$.

${ }^{25}$ Vgl. J. Leffers, op. cit.

${ }^{26}$ Vgl. G. Rosemann, Funny German Werbung. Die größten Blackouts deutscher Werbetexter, Bild online 2012, http://www.bild.de/ratgeber/2012/denglisch/werbung-werbetexter-fehler-blackout-robert-tonks2253279.bild.html (Zugriff am 20.02.2013).

${ }^{27}$ Vgl. ibidem.
} 
3) Hat ein verstandener Slogan einen positiven Einfluss auf die Einstellung zur Werbeanzeige?

4) Sind englische Werbeslogans erfolgreicher als die deutschen Werbeslogans?

\subsection{Befragte Personengruppen}

Die Befragung wurde im Zeitraum zwischen Januar und März 2014 realisiert. Von den Befragten haben 75 Prozent aus Bielefeld oder der näheren Umgebung, rund 20 Prozent aus München und 5 Prozent aus dem restlichen Bundesgebiet gestammt. Die Zielpersonen wurden per E-Mail oder persönlich zur Teilnahme an der Befragung eingeladen.

Insgesamt wurden 40 Personen befragt und allen wurden Fragen gestellt, wie sie bestimmte englische Werbeslogans verstehen. Die Befragten repräsentierten zwei Altersgruppen. In der ersten Personengruppe (20 Personen) wurden Jugendliche im Alter zwischen 17 und 22 Jahren befragt. Zu dieser Gruppe gehörten hauptsächlich Kollegiaten des Oberstufenkollegs an der Universität Bielefeld sowie Studenten der Universität Bielefeld. Die zweite Befragtengruppe dagegen haben Erwachsene im Alter von 25 bis 40 Jahren gebildet. Die meisten Befragten dieser Gruppe waren berufstätige Frauen und Männer mit Hochschulausbildung. Alle Befragten haben die Fragen, was die englischen Slogans für sie bedeuten, schriftlich oder mündlich beantwortet.

\subsection{Untersuchte Werbeslogans}

Für die Untersuchungen sind vier Werbeslogans in englischer Sprache ausgewählt worden, die in Deutschland populär sind. In den Slogans geht es um Produkte verschiedener Branchen wie Kosmetikkonzerne, Fluggesellschaften, Fast Food Restaurants und Automarken.

Die gleichen Werbeslogans wurden im Jahre 2009 von der Süddeutschen Zeitung in einer Leserumfrage untersucht. Die Leser haben damals überlegt, was die Slogans in der englischen Sprache tatsächlich bedeuten könnten. Die Ergebnisse dieser Umfrage zeigen deutlich, dass die Ausdrücke nicht verstanden werden.

Der erste englische Werbeslogan lautet „Come in and find out“ der Parfümerie Douglas, die fast nur englische Slogans auf dem deutschen Markt hat.

Eine mögliche Übersetzung dieses Slogans nach der Umfrage der „Süddeutschen Zeitung“ lautet, ,Komm rein und finde wieder raus“. ${ }^{28}$

Der zweite von mir gewählte und auch in der Befragung aus dem Jahre 2009 berücksichtigte Werbeslogan „There's no better way to fly“ der Fluggesellschaft Lufthansa wurde im Jahre 2000 eingeführt und ist bis heute erhalten geblieben.

Auf Deutsch könnte dieser Slogan nach der Meinung der Zeitungsleser lauten „Schneller kann man nicht entlassen werden“.29

Der Slogan der Fast Food-Kette Burger King „Have it your way“, der den dritten Platz in meiner Befragung hat, wurde schon vor ein paar Jahren in Deutschland eingeführt.

28 Vgl. Süddeutsche.de, http://www.sueddeutsche.de/leben/englische-werbeslogans-komm-rein-und-finde-raus1.141830 (Zugriff am 06.12.13).

${ }^{29}$ Vgl. Süddeutsche.de, http://www.sueddeutsche.de/leben/englische-werbeslogans-komm-rein-und-finde-raus1.141830-2 (Zugriff am 06.12.13). 
Die deutsche Version dieses Slogans nach den Zeitungslesern könnte man auf folgende Art und Weise übersetzen ,Nimm's mit auf den Heimweg“6. ${ }^{30}$

Vierter und zugleich letzter Werbeslogan meiner Befragung der Automarke Ford lautet „Ford - feel the difference“ und auf Deutsch „Ford - fühle den Streit“. ${ }^{31}$ Diese Übersetzung des Werbeslogans der Automarke Ford hat die von der „Suddeutschen Zeitung" durchgeführte Umfrage ergeben.

\section{ERGEBNISSE DER BEFRAGUNG UND SCHLUSSFOLGERUNGEN}

Die hier durchgeführten Umfragen zeigen andere Ergebnisse als die von 2009 der Süddeutschen Zeitung. Beide Gruppen von Befragten haben ähnlich geantwortet und die englischen Werbeslogans gut verstanden. Die erste befragte Personengruppe, also Jugendliche und Studenten, die die englische Sprache schon in der Schule als erste Fremdsprache gelernt haben, haben die Fragen u.a. auf folgende Art und Weise beantwortet:

- Wie verstehst Du diese englischen Werbeslogans?

„Come in and find out" von der Parfümerie Douglas:

1. „Komm herein und finde es heraus.“

2. „Komm her und lass dich überraschen.“

„There's no better way to fly“ von der Fluggesellschaft Lufthansa:

1. „Es gibt keinen besseren Weg zu fliegen als mit dieser Airline.“

2. „Man kann nicht besser fliegen (eine bessere Airline wählen).“

„Have it your way“ von der Fast Food-Kette Burger King:

1. „Mach es, wie du es willst. Ich entscheide, ob ich einen Burger genießen darf!“”

2. "Mach das auf deine eigene Art.“

„Ford - feel the difference“" von der Automobilmarke Ford:

1. und 2. „Ford - fühle den Unterschied.“

Die zweite Personengruppe im Alter zwischen 25 und 40 hat dieselben Fragen folgendermaßen beantwortet:

- Wie verstehen Sie diese englischen Werbeslogans?

„Come in and find out“ von der Parfümerie Douglas:

1. „Komm rein und finde es heraus. Also, komm in unser Geschäft und finde heraus, was es dort zu kaufen gibt.“

2. „Komm herein und finde raus, was dir gefällt.“

„There's no better way to fly“ von der Fluggesellschaft Lufthansa:

1. „Es gibt keinen besseren Weg zu fliegen. Also, Lufthansa ist die beste Möglichkeit, um zu reisen.“

2. „Es gibt keinen besseren Weg, um zu fliegen.“

„Have it your way“ von der Fast Food-Kette Burger King:

1. „Mach es auf deine Weise. Also, bei Burger King gibt es für jeden Geschmack das Passende."

2. „Hab es so wie du es magst.“

„Ford - feel the difference“"von der Automarke Ford:

30 Vgl. Süddeutsche.de, http://www.sueddeutsche.de/leben/englische-werbeslogans-komm-rein-und-finde-raus1.141830-3 (Zugriff am 06.12.13).

31 Vgl. Süddeutsche.de, http://www.sueddeutsche.de/leben/englische-werbeslogans-komm-rein-und-finde-raus1.141830-7 (Zugriff am 06.12.13). 
1. „Ford - Fühl den Unterschied. Also, erlebe beim Fahren den Unterschied zwischen Ford und anderen Automobilherstellern."

2. „Ford - fühle den Unterschied.“

Die Antworten machen deutlich, dass beide befragten Personengruppen diese Slogans korrekt verstanden haben.

Man kann davon ausgehen, dass das Verständnis des Werbeslogans von Alter und Schulbildung abhängig ist. Die befragen Personen haben ein Studium abgeschlossen oder studieren gegenwärtig. Englische Werbeslogans sind also kein Problem für ausgebildete Personengruppen. Auch die Einstellung zur Werbeanzeige ist er eher positiv als negativ. So kann man weiterhin annehmen, dass englische Werbeslogans so erfolgreich wie die deutschen Werbeslogans sind.

Geplant sind weitere Untersuchungen, um nächste Personengruppen zu befragen und dabei verschiedene Alters- und Sozialgruppen zu berücksichtigen. Die entstandenen Datenmengen lassen weitgreifende Schlussfolgerungen daraus ziehen.

Wenn man heutzutage einen Blick in Magazine wirft, Börseninformationen liest, in Kunden- oder Mitarbeiterzeitschriften blättert oder andere Medien in Deutschland verfolgt, stößt man häufig auf englische Begriffe und Wendungen, also Anglizismen. Das Englische ist auch die dominierende Sprache in der Werbung geworden.

Resümierend nach der Durchführung der Befragung muss man sagen, dass die englischen Werbeslogans für die Deutschen verständlich sind und keiner Erklärung bedürfen, da die meisten Deutschen die englische Sprache als erste Fremdsprache in der Schule lernen und in dieser Sprache problemlos kommunizieren können.

\section{LITERATURVERZEICHNIS}

[1] Adler M., Form und Häufigkeit der Verwendung von Anglizismen in deutschen und schwedischen Massenmedien, 2., neu bearbeitete Fassung, Jena 2004, http://www.dbthueringen.de/servlets/DerivateServlet/Derivate-3386/Adler.txt.

[2] Androutsopoulos J.K., Sprachwahl im Werbeslogan. Zeitliche Entwicklung und branchenspezifische Verteilung englischer Slogans in der Datenbank von slogans.de, Hannover 2004, S. 4, http://www.mediensprache.net/networx/networx-41.pdf.

[3] Burmasova S., Empirische Untersuchung der Anglizismen im Deutschen am Material der Zeitung Die WELT (Jahrgänge 1994 und 2004), Bamberger Beiträge zur Linguistik, University of Bamberg Press, Bamberg 2010, S. 10-11.

[4] Busse U., Anglizismen im Duden. Eine Untersuchung zur Darstellung englischen Wortguts in den Ausgaben des Rechtschreibdudens von 1880-1986, Tübingen 1993, (Reihe Germanistische Linguistik 139).

[5] Busse U., Typen von Anglizismen: von der heilago geist bis Extremsparing - aufgezeigt anhand ausgewählter lexikographischer Kategorisierungen, [In:] Hrsg. G. Stickel, Neues und Fremdes im deutschen Wortschatz. Aktueller lexikalischer Wandel. (Institut für deutsche Sprache, Jahrbuch 2000, Berlin 2001, S. 131-155.

[6] Carstensen B., Englische Einflüsse auf die deutsche Sprache nach 1945, Heidelberg 1965, (Beihefte zum Jahrbuch für Amerikastudien 13), S. 30.

[7] Czech-Rogoyska A., Denglisch - czas anglicyzmów w języków niemieckim, „Lingua legis” 2002/10, S. 53-63.

[8] Denglisch 4ever, http://www.denglisch4ever.de/autoframes.html?/top10.html.

[9] Duden-Deutsches Universalwörterbuch, Mannheim 2001, S. 388. 
[10] Herrmann L., Die zehn meistgebrauchten Werbewörter; (Quelle: Slogometer), http://www.wuv.de/marketing/die_zehn_meistgebrauchten_werbewoerter.

[11] Kick I., Die Wirkung von Anglizismen in der Werbung. „Just do it“ oder lieber doch nicht?, IFB Verlag, Paderborn 2004.

[12] Leffers J., Denglisch in der Werbung: Komm rein und finde wieder raus, Spiegel online. Unispiegel. 2004, http://www.spiegel.de/unispiegel/wunderbar/denglisch-in-der-werbungkomm-rein-und-finde-wieder-raus-a-310548.html.

[13] Meder K., Anglizismen in der deutschen Werbesprache: Untersucht anhand ausgewählter Frauen- und Männerzeitschriften, Berlin 2006.

[14] Rosemann G., Funny German Werbung. Die größten Blackouts deutscher Werbetexter, Bild online 2012, http://www.bild.de/ratgeber/2012/denglisch/werbung-werbetexter-fehlerblackout-robert-tonks-2253279.bild.html (Zugriff am 20.02.2013).

[15] Sowinski B., Werbung, Tübingen 1998, S. 59.

[16] Spiegel online. Wirtschaft, Werbung wieder deutsch: „Überleben Sie die Fahrt in unserem Auto “, 2004, http://www.spiegel.de/wirtschaft/werbung-wieder-deutsch-ueberleben-sie-diefahrt-in-unserem-auto-a-287098.html.

[17] Süddeutsche Zeitung, Süddeutsche.de, http://www.sueddeutsche.de/leben/englischewerbeslogans-komm-rein-und-finde-raus-1.141830-3.

[18] Tettenhammer Ch., Was ist ein Anglizismus? oder: Denglisch für Beginner, http://www.sprachenlernen24-blog.de/sprachen-der-welt-was-ist-ein-anglizismus/.

[19] Tuma Th., Die Sense stimuliert, „Spiegel“ 2003/38, S. 87.

[20] Verein Deutsche Sprache, Denglisch. Deutsch oder Denglisch?, http://www.vds-ev.de/agdenglisch-thema.

[21] Wächter A., Deutsche Sprache im 18. Jahrhundert - wie spiegeln sich kommunikative Verhältnisse in den Stillehrbüchern dieser Zeit wider?, Institut für Deutsche Sprache, Mannheim 1992, S. 89.

[22] Wirth K., Der Verein Deutsche Sprache. Hintergrund, Entstehung, Arbeit und Organisation eines deutschen Sprachvereins. Bamberger Beiträge zur Linguistik, University of Bamberg Press, Bamberg 2010, S. 157-163.

[23] Zindler H., Anglizismen in der deutschen Pressesprache nach 1945, Diss. Kiel 1959, S. 2. Zitiert nach: U. Busse, Anglizismen im Duden. Eine Untersuchung zur Darstellung englischen Wortguts in den Ausgaben des Rechtschreibdudens von 1880-1986, Tübingen 1993, (Reihe Germanistische Linguistik 139), S. 15.

\section{DENGLISCH IN THE LANGUAGE OF ADVERTISING - THE SURVEY ON THE DEGREE OF UNDERSTANDING OF THE ANGLICISMS IN GERMAN}

Each language that is alive is constantly changing. When we observe the contemporary linguistic tendencies we get the impression that languages exchange words, which results not only in the inclusion of specific foreign words into our native languages, but also in the assimilation of the new lexical items. The phenomenon of English words entering the German language is so intense that consequently we get a mixture of these two languages which is referred to as Denglisch.

The discussed phenomenon is increasingly present both in the specialist language varieties and in the common German. Advertising belongs to the areas, where we get a lot of examples of Denglisches: job positions, functions of the employees in the said field and advertising spots. The language of advertising is particularly rich in anglicisms. Not only do many of the German advertising slogans include anglicisms, but they are translated into 
English as a whole. Bearing in mind the ubiquity of the said phenomenon Denglisch has attracted a lot of attention in Germany in the recent years.

This paper presents the results of the study conducted by the author which aimed at confirming how far the German slogans are understandable for the German language users. The study was carried out in Germany, in two age groups on the basis of the four, randomly selected advertisements operating on the German market. Additionally, the paper presents some main notions related to the topic in point, that is anglicism, advertising slogan and Denglisch.

Keywords: Denglisch, language, anglicism, advertising slogan, advertisement

\section{DENGLISCH W JEZZYKU REKLAM - ANKIETA BADAJĄCA STOPIEŃ ZROZUMIENIA ANGLICYZMÓW W JEZYKU NIEMIECKIM}

W każdym żyjącym języku zachodzą stale zmiany. Obserwując współczesne tendencje językowe, można odnieść wrażenie, że języki wymieniają się słowami, co skutkuje nie tylko przeplataniem języka ojczystego obcobrzmiącymi zapożyczeniami, lecz także asymilacją nowego słownictwa. Przykładem takich zapożyczeń są stosowane w języku niemieckim anglicyzmy. Przenikanie anglicyzmów do języka niemieckiego jest tak intensywne, iż dochodzi coraz częściej do pomieszania tych dwóch języków oraz do powstania zjawiska Denglisch - tworu językowego, którego nazwa jest połączeniem słów - Deutsch i Englisch. Zjawisko to jest coraz częściej zauważalne zarówno w specjalistycznych odmianach języka niemieckiego jaki i w języku potocznym. Jedną z dziedzin, w której Denglisch jest szczególnie widoczny i popularny, jest reklama. Począwszy od stanowisk i funkcji pracowników tej branży, a kończąc na spotach reklamowych, język reklamy jest niezwykle bogaty w anglicyzmy. W wielu przypadkach, niemieckie slogany reklamowe nie tylko zawierają anglicyzmy, lecz są w całości tłumaczone na język angielski. Zważywszy na jego wszechobecność, zjawisku Denglisch poświęca się w Niemczech wiele uwagi. Dla jednych Denglisch jest znakiem otwartości na świat, a przede wszystkim dowodem rozwoju języka, podczas gdy inni postrzegają go jako zagrożenie dla języka niemieckiego. W niniejszym artykule autorka przedstawia wyniki własnych badań mających na celu rozpoznanie, czy slogany reklamowe w języku angielskim są zrozumiałe dla odbiorców niemieckich. Badania zostały przeprowadzone w Niemczech w dwóch grupach wiekowych na przykładzie czterech losowo wybranych reklamach występujących na rynku niemieckim. W artykule przedstawiono również główne pojęcia związane z tematem czyli anglicyzm, slogan reklamowy i Denglisch oraz scharakteryzowano niemieckie slogany reklamowe z pod kątem najczęściej stosowanych w nich słów.

Słowa kluczowe: Denglisch, język, anglicyzmy, slogan reklamowy, reklama

\section{DOI: $10.7862 /$ rz.2014.mmr.30}

Tekst złożono w redakcji: maj 2014

Przyjęto do druku: wrzesień 2014 